Sharif University of Technology
Scientia Iranica
SCIENTIA
I RAN I CA
http://scientiairanica.sharif.edu

\title{
Probabilistic model of unsaturated slope stability considering the uncertainties of soil-water characteristic curve
}

\author{
A. Johari*, A. Hooshmand Nejad, and S. Mousavi \\ Department of Civil and Environmental Engineering, Shiraz University of Technology, Shiraz, Iran. \\ Received 15 November 2016; received in revised form 7 January 2017; accepted 25 February 2017
}

\author{
KEYWORDS \\ Unsaturated soils; \\ Slope stability; \\ Soil-water \\ characteristic curve; \\ Artificial intelligence; \\ Probabilistic model.
}

\begin{abstract}
Many soil slopes are unsaturated and their failure can be a major cause of damage to structures. Apart from soil properties, the Soil-Water Characteristic Curve (SWCC) is the backbone of any unsaturated slope analysis. Uncertainties of these effective parameters of unsaturated slopes make the probabilistic analysis more realistic, rather than deterministic. In this research, the stochastic analysis of unsaturated slope stability is carried out based on the simplified Bishop's method. The stochastic parameters are the input parameters of SWCC, in addition to effective internal angle of friction, effective cohesion, and unit weight of soil. Based on the collected results from hundreds of stochastic analyses, the probability of failure is presented as a three-dimensional surface. Finally, the probabilistic model is developed to model this surface and evaluate the probability of failure as a function of safety factor and its correlation of variation.

(C) 2018 Sharif University of Technology. All rights reserved.
\end{abstract}

\section{Introduction}

Many geotechnical structures, such as slopes, embankments, earth dams, retaining walls, and foundations, are usually located above the groundwater table; thus, their response involves layers with negative pore-water pressures, which are generally unsaturated. The effects of negative pore-water pressure are usually neglected in engineering practices because the groundwater table is assumed conservatively to be on the ground surface, or the soil above the water table surface is assumed to be dry in the sense that pore pressure is set to zero in the geotechnical analyses [1].

The success of any unsaturated analysis, such

\footnotetext{
*. Corresponding author.

E-mail addresses: johari@sutech.ac.ir (A. Johari);

a.hooshmandnejad@sutech.ac.ir (A. Hooshmand Nejad);

s.mousavi@sutech.ac.ir (S. Mousavi)
}

doi: $10.24200 /$ sci.2017.4202 as unsaturated slope stability, greatly depends on the input data, such as SWCC, that defines the relationship between the amounts of water in soil (i.e., gravimetric water content, volumetric water content or degree of saturation and matric suction [2]).

Several stochastic analyses of unsaturated slope were presented in literature. Bergardo and Anderson [3] and Gui et al., 2000 [4] considered the influence of uncertainties associated with pore pressures on slope reliability. The results of this reliability analysis are expressed in terms of reliability index. Reliability computations performed for the slope show that failure zones are better captured by the reliability index than the conventional factor of safety [5]. Wolff [6] suggested that a reliability index of 3 for routine slopes and 4 for critical slopes is acceptable. The U.S. Army Corps of Engineers [7] recommended the characterization of safety based on reliability index for implementation in geotechnical engineering practice.

The influence of the spatial variability of the saturated hydraulic conductivity and the nature of 


$$
F=\frac{\sum\left[c^{\prime} \beta R+\left\{N-u_{w} \beta \frac{\tan \phi^{b}}{\tan \phi^{\prime}}-u_{a} \beta\left(1-\frac{\tan \phi^{b}}{\tan \phi^{\prime}}\right)\right\} R \tan \phi^{\prime}\right]}{\sum W x-\sum N f},
$$

where:

$$
\begin{aligned}
& N=\frac{W-\left(X_{R}-X_{L}\right)-\frac{c^{\prime} \beta \sin \alpha}{F}+\frac{u_{a} \beta \sin \alpha}{F}\left(\tan \phi^{\prime}-\tan \phi^{b}\right)+u_{w} \frac{\beta \sin \alpha}{F} \tan \phi^{b}}{m_{\alpha}}, \\
& m_{\alpha}=\left\{\cos \alpha+\frac{\sin \alpha \tan \phi^{\prime}}{F}\right\} .
\end{aligned}
$$

Box I

triggering mechanisms on the risk of rainfall-induced landslides (for an infinite slope) was studied [8]. It was shown that a probabilistic framework can be used to efficiently consider various failure patterns caused by spatial variability of hydraulic conductivity in rainfall infiltration assessment for a shallow infinite slope [9]. Zhang et al. [10] developed some spreadsheet templates for their suggested method to predict rainfall-induced slope.

The first aim of this paper is to study the influence of uncertainty of SWCC, in addition to soil properties' uncertainties in unsaturated slope stability analysis. For this purpose, in different steps, uncertainties of SWCC and soil properties are considered and studied in slope stability analysis. Moreover, in this research, the probability of failure is studied as a function of safety factor and its correlation of variation because it is highly possible that two slopes with the same soil properties and geometries can have different probabilities of failure due to differences in variability of the soil properties. Therefore, the probability of failure is investigated as a three-dimensional (3D) surface. The presented surface for probability of failure is modelled by Gene Expression Programming (GEP) as a powerful artificial intelligence method to predict equations without any prior assumption.

\section{Analysis method for unsaturated slope stability}

It is well known that suction of unsaturated soils has significant influence on any analysis of these soils due to the effect of basic mechanical properties of soils such as shear strength. In this study, the General Limit Equilibrium (GLE) method is used to analyse the stability of slope. The GLE method provides a general theory wherein other methods can be viewed as special cases [11]. The Factor of Safety (FS) with respect to moment equilibrium is calculated through Eq. (1) [2] as shown in Box I. The parameters used in
Eqs. (1) to (3) (shown in Box I) are defined as follows:

$W \quad$ The total weight of the slice of width $b$ and height $h$

$N \quad$ The total normal force on the base of the slice

$e \quad$ The horizontal inter-slice normal forces ( $L$ and $R$ subscripts designate the left and right sides of the slice, respectively)

$X \quad$ The vertical inter-slice shear forces $(L$ and $R$ subscripts designate the left and right sides of the slice, respectively)

$R \quad$ The radius for a circular slip surface or the moment arm associated with the mobilized shear force, for any shape of slip surface

$f \quad$ The perpendicular offset of the normal force from the centre of rotation The horizontal distance from the centreline of each slice to the centre of rotation

$\alpha \quad$ The angle between the tangent to the centre of the base of each slice and the horizontal

$\beta \quad$ The length of the base of a slice

$\sigma_{n} \quad$ Total normal stress

$u_{a} \quad$ Pore-air pressure

$u_{w} \quad$ Pore-water pressure

$c^{\prime} \quad$ Effective cohesion

$\phi^{\prime} \quad$ Effective internal friction angle

$\tan \phi^{b} \quad$ Angle related to the change in shear strength with respect to a change in suction

The GLE method can be used to simulate various methods of slices using the appropriate inter-slice force 


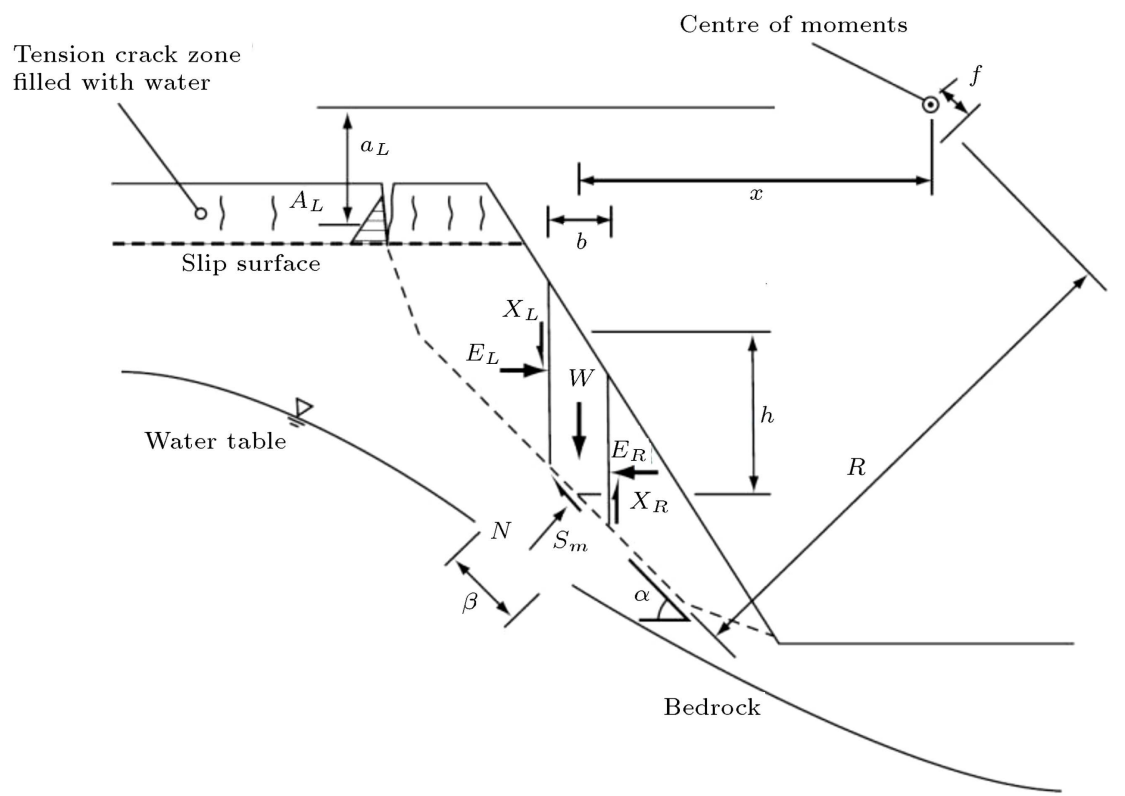

Figure 1. Forces acting on a slice through a sliding mass [2].

assumption [2]. In this study, the simplified Bishop's method is considered in unsaturated slope stability analysis (Figure 1). The simplified Bishop's method assumes circular slip surface and zero inter-slice shear forces in slope stability analysis [12].

\section{Soil-water characteristic curve}

In this research, an artificial intelligence model of SWCC is used in the analyses. From previous works on the topic, it is well understood that the SWCC is dependent on the grain size distribution, soil density, suction and water content of the soil. Hence, any of the SWCC parameters and/or any combination of these parameters may be considered as appropriate candidates for inputs of the model. The model, namely GEP, has acceptable accuracy to predict the SWCC [13]. GEP model uses the soil properties as inputs to predict SWCC and does not use the curvefitting parameters. The GEP model for predicting SWCC is as follows [13]:

$$
\begin{aligned}
\omega= & \frac{-1}{S+2 \mathrm{Cl}-2.202 \frac{S^{4}}{\mathrm{Cl}^{2}}-7.285} \\
& -\omega_{0}\left(S+0.062(\mathrm{Si}+e)^{2}-1\right)
\end{aligned}
$$

where $e$ is initial void ratio, $\omega_{0}$ is initial water content, $S$ is defined as $\log$ [suction $(\mathrm{kPa}) / \mathrm{pa}$ ] where pa is atmospheric pressure (taken as $100 \mathrm{kPa}$ ), $\mathrm{Cl}$ and $\mathrm{Si}$ are clay and silt contents (\%), and $\omega$ is predicted gravimetric water content.

Table 1 shows the correlation coefficient and error values of this model to predict the SWCC based on SoilVision (2002) databank [14], which contains more than 180 pressure plate tests results performed on clay, silty clay, sandy loam, and loam soil. In this table, Average Relative Error (ARE), the Mean Squared Error (MSE) and $R^{2}$ are defined by:

$$
\begin{aligned}
& \mathrm{MSE}=\frac{1}{N} \sum_{i=1}^{N}\left(A_{i}-P_{i}\right)^{2}, \\
& \mathrm{ARE}=\frac{1}{N} \sum_{i=1}^{N}\left|\frac{A_{i}-P_{i}}{A_{i}}\right| \times 100,
\end{aligned}
$$

where $A_{i}$ is actual value for the $i$ th data, $P_{i}$ is predicted output data for the $i$ th data, and $N$ is total number of data available in the database. Moreover, $R^{2}$ is correlation coefficient (square of the Pearson product moment correlation coefficient).

One of the advantages of the SWCC model for reliability and stochastic analysis is that it has a specific equation for SWCC prediction. Therefore,

Table 1. Performance of GEP model [13].

\begin{tabular}{ccccccccc}
\hline & \multicolumn{3}{c}{ Training data } & & \multicolumn{3}{c}{ Testing data } \\
\cline { 1 - 3 } \cline { 6 - 9 } GEP model & ARE $(\%)$ & MSE & $R^{2}$ & & ARE (\%) & MSE & $R^{2}$ \\
& 25.59 & 0.0014 & 0.94 & & 28.73 & 0.0014 & 0.94 \\
\hline
\end{tabular}


there is no undetermined variable, such as the curve fitting parameters, that could generate some difficulties (such as managing the proper correlation between curve fitting parameters). On the other hand, the input parameters of this model are based on grain size distribution curve and inherent soil properties, which are absolutely well known among geotechnical engineers.

The comprehensive explanation of this method is available in [13]; however, as an example, Figure 2 shows the prediction of SWCC by GEP model for a typical soil sample of databank with the following properties:

- Void ratio: 0.71;

- Initial water content: $26.89 \%$;

- Clay content 28.71\%;

- Silt content: $63.57 \%$.

For this soil sample, the complete procedure for calculating SWCC using GEP model is presented in the Appendix.

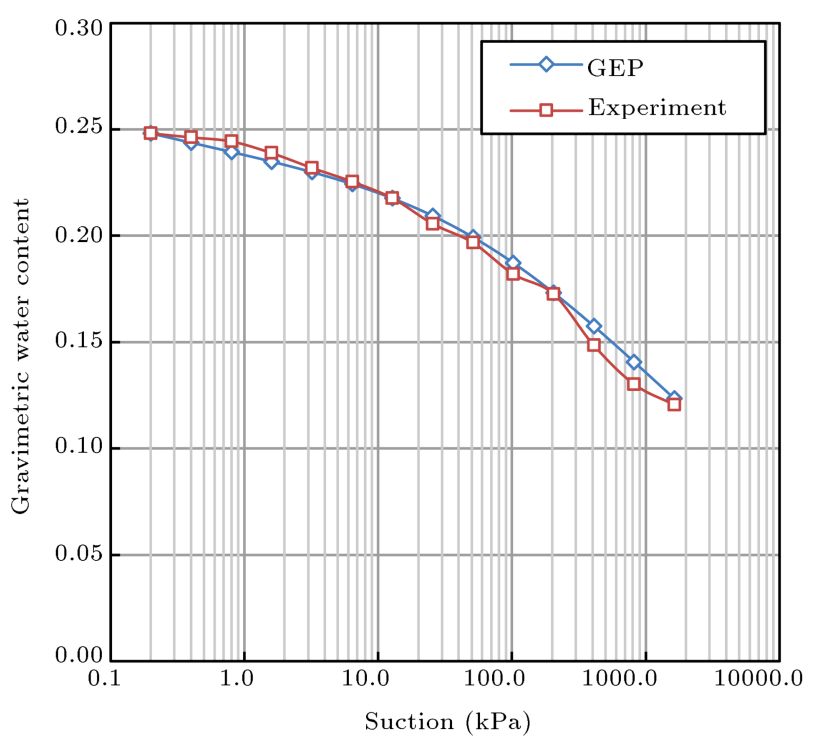

Figure 2. A typical SWCC prediction of GEP model.

\section{Deterministic unsaturated slope stability analysis}

To initiate unsaturated slope stability, a typical slope is analysed using simplified Bishop's method. Table 2 shows the arbitrary soil properties, selected from SoilVision databank [14]. For this purpose, a typical slope (Figure 3) with length of $20 \mathrm{~m}$ and a gradient of $2(\mathrm{H}): 1(\mathrm{~V})$ is used. The suction is controlled by the water content of the soil layers. The water table is assumed to be at heel of slope. Determining the water content or pore water pressure at slope using various approaches is well studied in the literature [15-17]. However, in this research, some simplified assumptions of water content distribution are considered in the analysis. It is assumed that suction linearly increased from zero (at water table) to maximum value at the ground surface (A-B level). The suction at the ground surface is obtained through water content of slope surface and selected soil properties using GEP model of SWCC. The water content of the soil at the ground surface is assumed to be a logical value of $24.5(\%)$, and the pore air pressure is assumed atmospheric $\left(u_{a}=\right.$ $0)$. Other mechanical and geometrical parameters are given in Table 3 .

In order to evaluate the slope stability, a computer program was coded in MATLAB based on the simplified Bishop's method. MATLAB is a powerful multi-paradigm numerical computing environment and fourth-generation programming language. MATLAB allows matrix manipulations, plotting of functions and data, implementation of algorithms, creation of user interfaces, and interfacing with programs written in other languages, including C, C++, C\#, Java, Fortran, and Python.

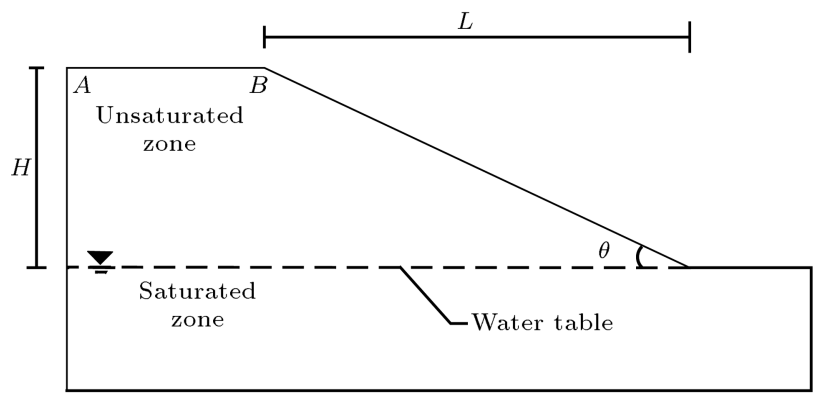

Figure 3. Geometry of slope.

Table 2. Soil properties used as input parameters of SWCC model.

\begin{tabular}{cccc}
\hline Initial void ratio & Initial water content & Clay content & Silt content \\
\hline 0.81 & 30.12 & 0.50 & 0.40 \\
\hline
\end{tabular}

Table 3. Properties of slope used in slope stability analysis.

\begin{tabular}{cccccc}
\hline $\boldsymbol{L}(\mathbf{m})$ & $\boldsymbol{H}(\mathbf{m})$ & $\boldsymbol{c}^{\prime}(\mathbf{k P a})$ & $\phi^{\prime}($ degree $)$ & $\phi^{\mathbf{b}}(\mathbf{d e g r e})$ & $\boldsymbol{\gamma}\left(\mathrm{KN} / \mathbf{m}^{\mathbf{3}}\right)$ \\
\hline 20 & 10 & 10 & 20 & 10 & 20 \\
\hline
\end{tabular}




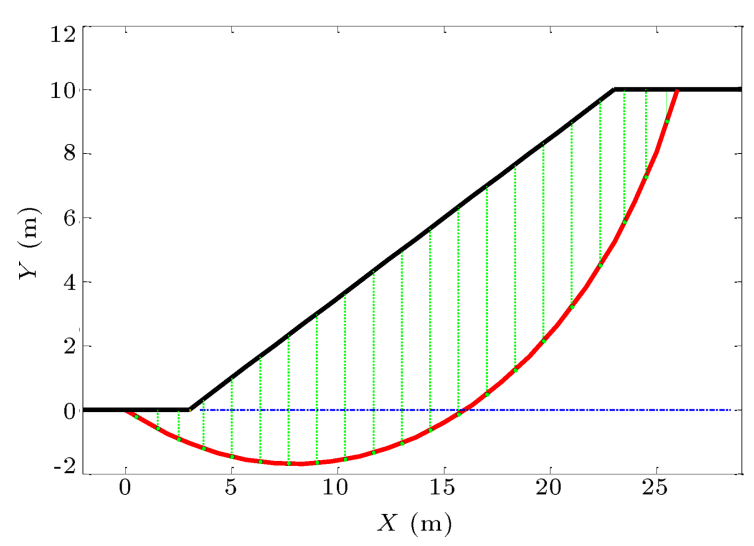

Figure 4. Calculated critical slip surface of the slope.

Figure 4 shows the critical slip surface of slope obtained from the Particle Swarm Optimization (PSO) algorithm. PSO is a computational method that optimizes a problem by iteratively trying to improve a candidate solution with regard to a given measure of quality. It solves a problem through a population of candidate solutions, dubbed as particles herein, and moving these particles around in the search-space according to simple mathematical formulae over the particle's position and velocity. Each particle's movement is influenced by its local best-known position; however, it is also guided toward the best-known positions in the search space, which are updated since better positions are found by other particles. It is expected that the swarm move toward the best solutions [18-20] .

Based on the conducted analysis, the minimum factor of safety related to critical slip surface of the slope is $\mathrm{FS}=1.52$. In this approach, using the artificial intelligence model of SWCC, there is no need for measuring negative pore water pressures above the groundwater table or performing pressure plate to access the SWCC.

\subsection{Verification of the coded programs}

There are various feasible alternatives to simplified Bishop's method. Other limit equilibrium methods, such as Ordinary, Janbu, and Morgenstern-Price methods, are among feasible alternatives. Moreover, numerical methods, such as Finite-Element Method, are the other options. However, the simplified Bishop's method is an acceptable method widely used in the literature due to its accuracy and simplicity. To clarify the efficiency of this method and the possible effect of it, the result of analysing the slope with this method is verified and compared with that of finiteelement method. For this purpose and to verify the accuracy of the developed code, the selected slope is also analysed by finite-element method, which is coded in MATLAB. Figure 5 shows its finite-element discretization. The safety factor of a slope by finiteelement method is obtained by generating the effective stress field due to gravity and suctions, followed by a

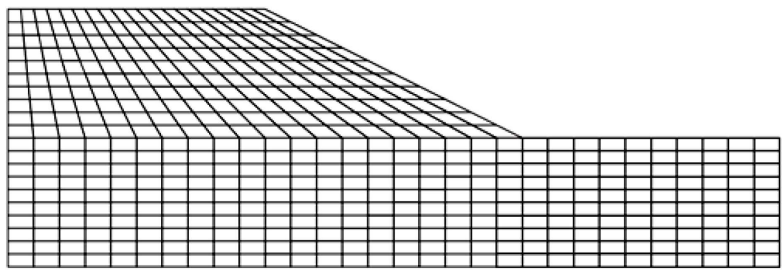

Figure 5. Finite-element discretization.

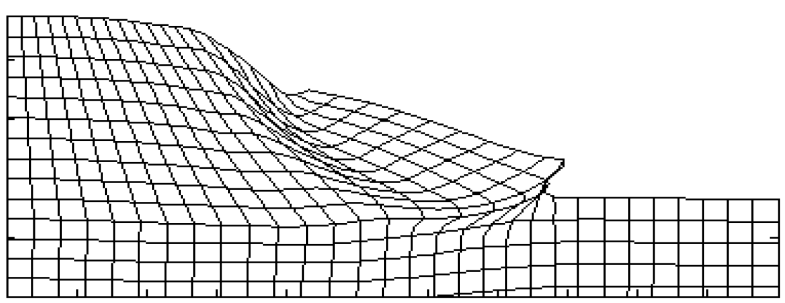

Figure 6. Failure mechanisms from finite-element analysis.

systematic reduction in shear strength parameters tan $\phi^{\prime}$ and $c^{\prime}$, until the algorithm is unable to satisfy both the Coulomb criterion and global equilibrium [21]. The obtained factor of safety from this method is FS $=1.53$. The failure mechanism from finite-element analysis is shown in Figure 6. The difference between the safety factor resulted from this approach (1.53) and that resulted from developed limit equilibrium MATLAB code in previous section (1.52) is less than $1 \%$; thus, the results are perfectly compatible.

\section{Stochastic unsaturated slope stability analysis}

To investigate the effect of SWCC uncertainties on the analysis of unsaturated slope stability, different conditions are specified and the analysis is performed for each of them separately. It can help have a better understanding of the influence of SWCC uncertainties on unsaturated slope stability. The determined conditions are as follows:

a) Considering uncertainties of $S W C C$ : In this condition, just the soil input parameters of SWCC have been defined as stochastic variables. The SWCC model uses some basic soil properties as the input parameters. The uncertainty of SWCC results from that of its input parameters; thus, they are set as stochastic parameters. For this purpose, a truncated normal probability distribution function was assumed for void ratio, initial water content, clay content, and silt content as presented in Table 4. The normal distribution is a very common continuous probability distribution. Normal distribution is so important in statistics and is often used in the literature because it is highly compatible with the uncertainty of soil properties. 


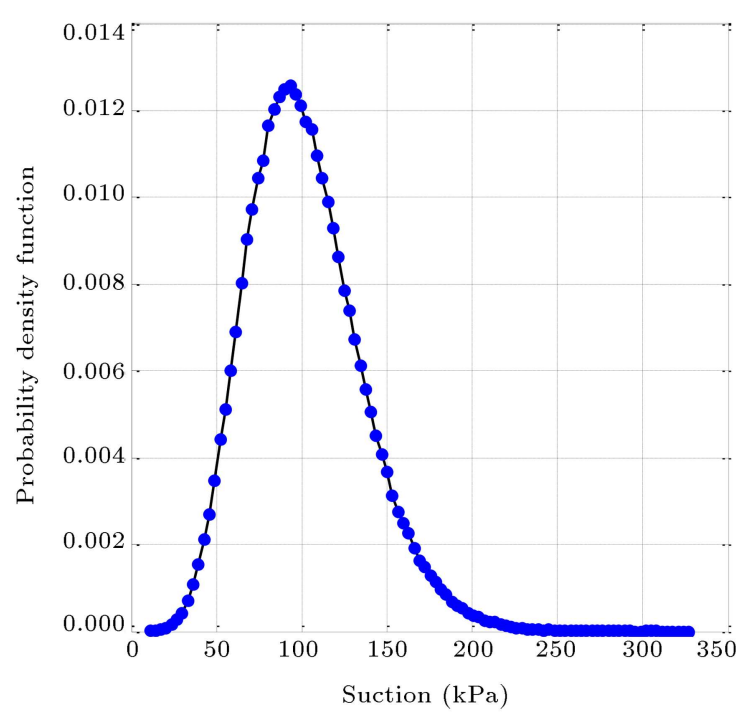

Figure 7. Probability density function of suction.

In fact, for each random series of stochastic inputs of Eq. (4), the equation is solved and the suction is achieved. However, in unsaturated slope stability analysis, the suction is needed (e.g., Eq. (6)). Therefore, the model was solved to extract dispersion of suction for specific water content based on stochastic input parameters. As a result, the probability density function (pdf) of suction as a stochastic parameter is determined (Figure 7 ).

The geometry and other parameters are considered the same as the deterministic analysis in a previous part. The result of probabilistic analysis of unsaturated slope stability is shown as pdf of factor of safety in Figure 8. The safety factor is continuously increasing from 1.46 , when the suction is $8 \mathrm{kPa}$ and the soil is almost saturated to 1.78 at the maximum suction, which generated $20 \%$ change in safety factor. However, considering other conditions in slopes, higher uncertainties in input parameters of SWCC and calculating suction in every single point of slopes may rise this difference considerably;

b) Considering uncertainties of SWCC and soil properties: In this part, the SWCC model's

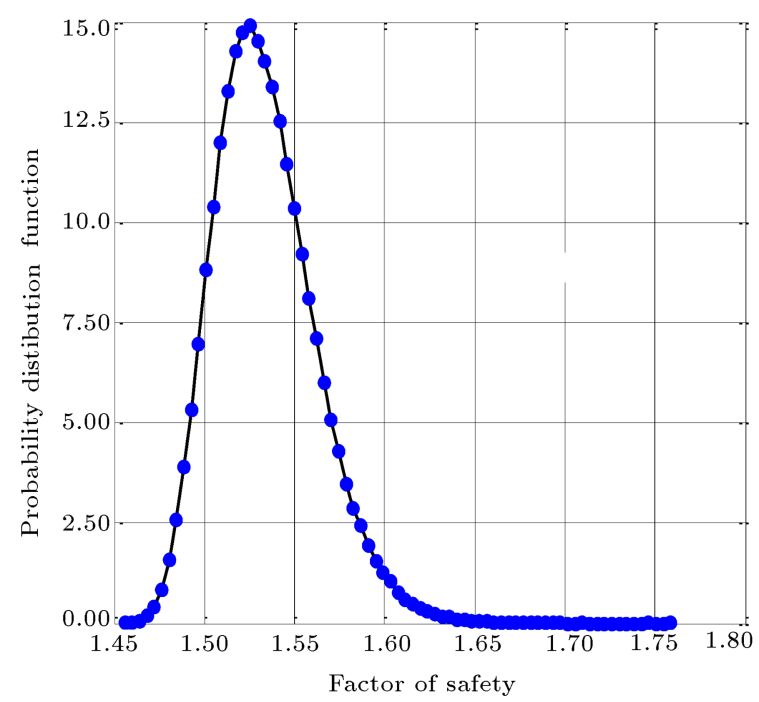

Figure 8. Probability density function of safety factor.

parameters (void ratio, initial water content, clay content, and silt content) and important mechanical properties of soils (effective cohesion, effective friction angle, and unit weight) are considered as stochastic parameters. The stochastic parameters with truncated normal probability distribution function are given in Table 4.

The results of this analysis are shown as a probability density function of factor of safety in Figure 9. On the other hand, deterministic analysis using the mean value of the stochastic parameters (as Section 4.1) shows that the safety factor of unsaturated slope is about 1.52. This demonstrates that the slope is stable; however, the probability of stability or failure is not specified. Although this safety factor is approximately the most probable value, it is probable to encounter less safety factors in this slope. Based on Figure 9, the probability of the presence of this safety factor value and less (FS $\leq 1.52$ ) is considered to be about $45 \%$. Furthermore, the safety factor, in which there is no possible value below it, is 2.26. Therefore, a designer can achieve a useful tool for engineering judgment. In fact, reliability assessment and engi-

Table 4. Stochastic parameters.

\begin{tabular}{cccccc}
\hline \multirow{4}{*}{ SWCC } & Parameter & Mean & Standard deviation & Maximum & Minimum \\
\hline \multirow{5}{*}{ Soil } & e & 0.810 & 0.020 & 0.830 & 0.790 \\
& $\omega_{0}(\%)$ & 30.120 & 0.753 & 30.873 & 29.367 \\
& $\mathrm{Cl}(\%)$ & 0.500 & 0.012 & 0.512 & 0.487 \\
& $\mathrm{Si}(\%)$ & 0.400 & 0.010 & 0.410 & 0.390 \\
\hline \multirow{3}{*}{${ }^{\prime}(\mathrm{kPa})$} & 10.000 & 2.000 & 12.000 & 8.000 \\
& $\phi^{\prime}($ degree $)$ & 20.000 & 2.000 & 22.000 & 18.000 \\
& $\gamma\left(\mathrm{kN} / \mathrm{m}^{3}\right)$ & 20.000 & 1.000 & 21.000 & 19.000 \\
\hline
\end{tabular}




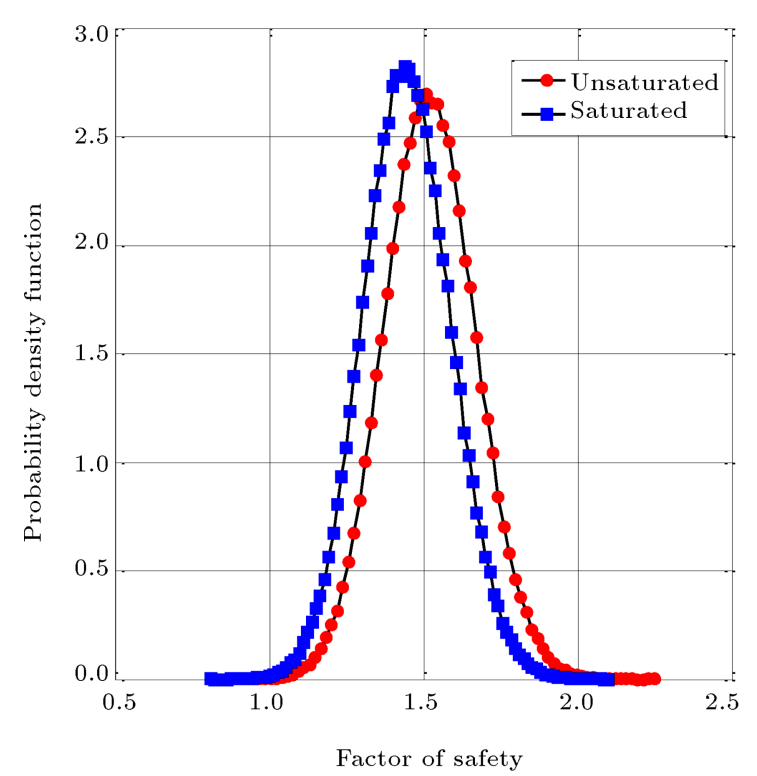

Figure 9. Probability density function of factor of safety in saturated and unsaturated slopes.

neering judgment are employed together to develop risk and decision analyses;

c) Considering uncertainties of soil properties in saturated state: At the last part, the analysis was repeated for the saturated condition of slope. In other words, the effects of suction and SWCC on slope stability are neglected. The pdf of safety factor related to this analysis is illustrated in Figure 9. In this case, the pdf shifted to the left and the minimum and maximum of the probable safety factor decreased to 2.1 and 0.81 , the same as the most probable safety factor, which declined to 1.44 .

However, the standard deviation of safety factor does not have a considerable difference in saturated and unsaturated analyses. It is 0.39 in unsaturated condition and 0.38 in saturated analysis. The mean of probable safety factors increased from 1.45 in saturated condition to 1.58 in unsaturated conditions.

\section{Probabilistic model development}

In order to develop a probabilistic model for unsaturated slope stability, several random series of input parameters, including correlation coefficient -0.5 between $c^{\prime}$ and $\phi^{\prime}$ [22] and the correlation coefficient 0.95 between $e$ and $\omega_{0}$ (resulted from real samples of SoilVision databank [14]), were considered. The correlation coefficient could be a value between -1 and 1 where 1 indicates a completely direct relation between two parameters; on the contrary, -1 represents a completely adverse relation. The correlation between $c^{\prime}$ and $\phi^{\prime}$ was well studied in the literature, and -0.5 was presented as an acceptable correlation coefficient between $c^{\prime}$ and $\phi^{\prime}$.

Moreover, in order to consider the possible influence of geometry and generalize the analyses, the slopes were analysed in various arbitrary geometries with slope angles of 30, 40, 50, and 60 degrees.

Probability of failure in slopes was studied in 2D and, then, 3D spaces. In the 2D form, the probability of failure was assessed with respect to the safety factor. However, in the 3D form, the Coefficient Of Variation (COV) of safety factor was added to the analysis.

\subsection{Modelling the probability of slope failure}

To develop a probabilistic model for unsaturated slope stability, the steps below were followed:

- Several random series of input parameters $c^{\prime}, \phi^{\prime}, \gamma$, $e, \omega_{0}, \mathrm{Cl}$, and $\mathrm{Si}$ were considered as mean values;

- A finite slope with arbitrary horizontal length $(L)$ and angle of slope $(\theta)$ was selected (the arbitrary slope angles of $30,40,50$, and 60 degrees were selected);

- The critical slip surface for each data series was obtained by the PSO technique;

- The uncertainty in the input parameters for each series of database was assessed;

- The pdf and cumulative distribution function (cdf) of factor of safety was determined for each data series;

- The probability of failure was computed from the cdf for each series (the cdf corresponded to FS $\leq 1$ );

- The factor of safety of each data series was calculated using the deterministic approach based on simplified Bishop's method (Eq. (1));

- The probability of failure and the related factor of safety from two previous steps were plotted with respect to each other. Figure 10 demonstrates the results for a number of data series.

The factor of safety cannot be a sufficient index for reliability analysis of slopes; moreover, as seen in Figure 10, the same safety factor may lead to various probabilities of failure. It is possible that two slopes with nominally identical soil properties and the same geometries can have different probabilities of failure due to differences in variability of the soil properties. The probability of failure with regard to the safety factor is not a 2D unique graph, and it is highly related to the variability of the safety factor too. Therefore, the probability of failure and the related factor of safety with respect to coefficient of variation of safety factor must be studied in a three-dimensional space. In this research, based on this phenomenon, the probabilities of failure regarding the safety factor at various COVs of 


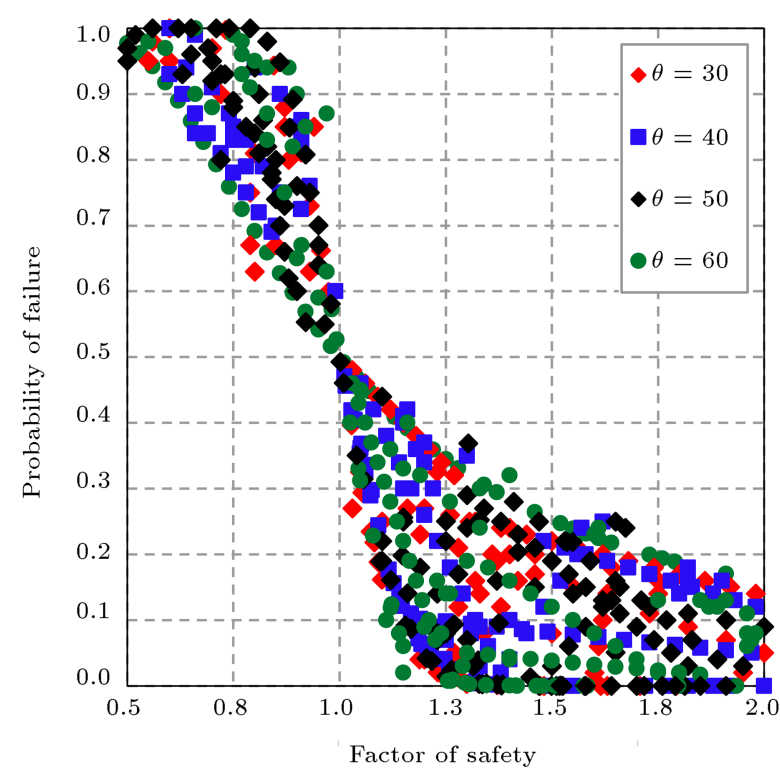

Figure 10. The probability of slope failure with respect to factor of safety.

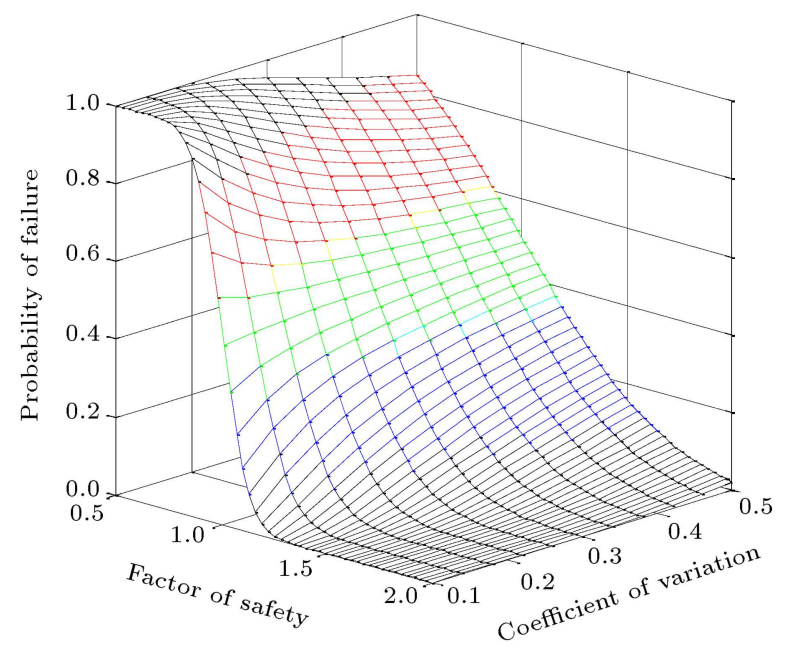

Figure 11. The 3D surface of probability of slope failure.

safety factor are calculated, and the results are plotted in Figure 11.

For a better understanding of Figure 11, it is plotted in a two-dimensional graph for some specific COVs. Figure 12 shows the probability of failure curve for various coefficients of variation from 0.1 to 0.5 . It is clear that, at FS $=1$, all of the curves pass through a common point, which corresponds to the probability of failure of $50 \%$. It can be divided into two parts: $F S<1$ and FS $>1$. For the safety factors less than 1 , the probability of failure decreased with an increase in COV. Contrarily, for the safety factors greater than 1, the probability of slope failure arises with increasing the COV. However, as a principle for each curve, the greater the factor of safety, the less probability of failure.

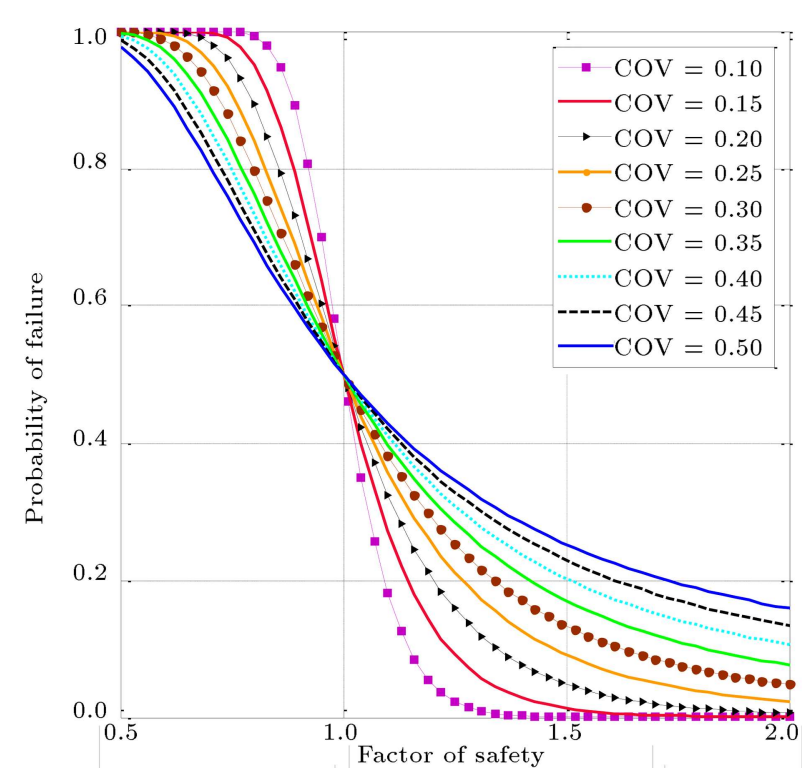

Figure 12. The probability of failure in various COVs of safety factor.

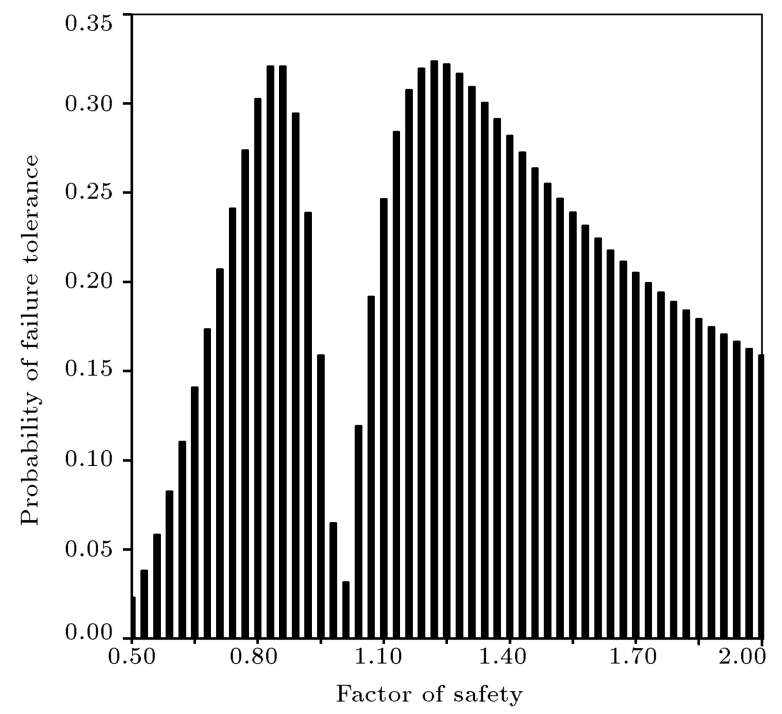

Figure 13. The probability of failure tolerance in various safety factors.

Moreover, the range of possible probabilities of failure varies in different safety factors. Figure 13 illustrates the tolerance of probability of failure in various safety factors between 0.5 and 2.0.

\subsection{Model development using GEP}

In this section, attempt is made to model the presented surface in Figure 10. The surface is rather complicated with an irregular curve which is not applicable to modelling by the curve-fitting approach; thus, a soft computing technique could be a suitable option. There have been many scientific efforts directed at applying soft-computing techniques, specially GEP 
to engineering [23-29]. The principal advantage of the GEP approach is its ability to generate powerful predictive equations without any prior assumption of the possible form of the functional relationship [30]. In this research, the GEP is employed as an artificial intelligence method to model the probability of failure surface and predict the probability of failure. This modelling covers the COV range from $10 \%$ to $50 \%$ based on literature $([31,32])$ and the range of practical safety factors, which are obviously FS $>1$. For this purpose, $75 \%$ of data series are selected randomly as training data for teaching the model and the rest as testing data for testing the developed model.

The GEP software [33] was used to perform symbolic regression using GEP and model the probability of failure. In GEP method, the values of setting parameters have huge influence on the fitness of the final output model [30]. Among these parameters, the most important ones are the number of genes, gene's head size, number of chromosomes, and the rate of genetic operators. This approach involved using different setting parameters and conducting runs in separate steps to find the suitable setting parameters. During each step, runs were carried out and the values of one of the above-mentioned parameters varied, whereas the values of the other parameters were kept constant. At the end of each run, the MSE for both training and testing sets was recorded in order to identify the values that give the least MSE [13]. The input parameters used in this modelling are presented in Table 5 .

One of the advantages of the GEP technique is that the relationship between the inputs of the model and the corresponding outputs is automatically constructed in the Expression Trees (ET) [13]. In this modelling, the appropriate final ET consists of two subtrees (ET1 and ET2) that are linked to each other by "addition" to produce the final model, presented in Figure 14. The depicted ETs in Figure 13 can be formulated easily into a mathematical equation as in Eq. (9). In this formula, the probability of failure is a function of deterministic safety factor and coefficient of variation of safety factor:

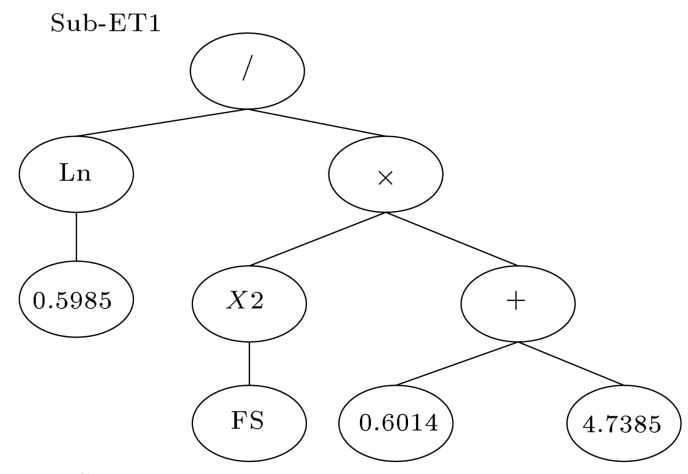

Sub-ET2

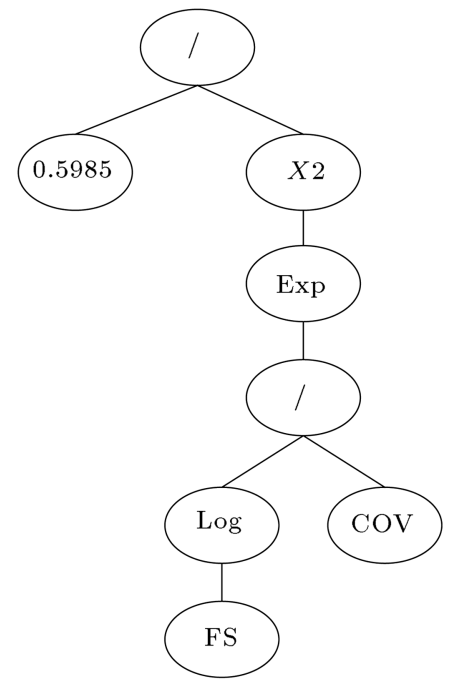

Figure 14. Expression tree of the proposed model.

$$
P_{f}=0.598 / \mathrm{FS}^{0.869 / \mathrm{COV}}-0.096 / \mathrm{FS}^{2},
$$

where $P_{f}$ is the probability of failure, FS is defined deterministic safety factor, and $\mathrm{COV}$ is coefficient of variation of safety factor.

Previous modelling of probability of failure for other geotechnical problems, such as liquefaction or seismic slope stability, studied the probability of failure just as a function of safety factor. However, the proposed model in this research adds the COV to the modelling. This can be more compatible with the

Table 5. Input parameters used for the GEP modelling.

\begin{tabular}{ll}
\hline Parameter & Achieved functions, values and rates \\
\hline Fitness function & MSE \\
Linking function & Addition $(+)$ \\
Function set &,,$+- \div, \times$, Exp, Log, Ln \\
Number of chromosomes & 30 \\
Number of genes & 2 \\
Gene head size & 5 \\
Recombination rate & 0.2 \\
Mutation rate & 0.044 \\
\hline
\end{tabular}


Table 6. Performance of GEP model.

\begin{tabular}{cccccccc}
\hline & \multicolumn{3}{c}{ Training data } & & \multicolumn{3}{c}{ Testing data } \\
\cline { 2 - 4 } \cline { 6 - 8 } GEP model & ARE (\%) & MSE & $R^{2}$ & & ARE $(\%)$ & MSE & $R^{2}$ \\
& 6.47 & $5.7 \times 10^{-5}$ & 0.99 & & 6.28 & $5.5 \times 10^{-5}$ & 0.99 \\
\hline
\end{tabular}

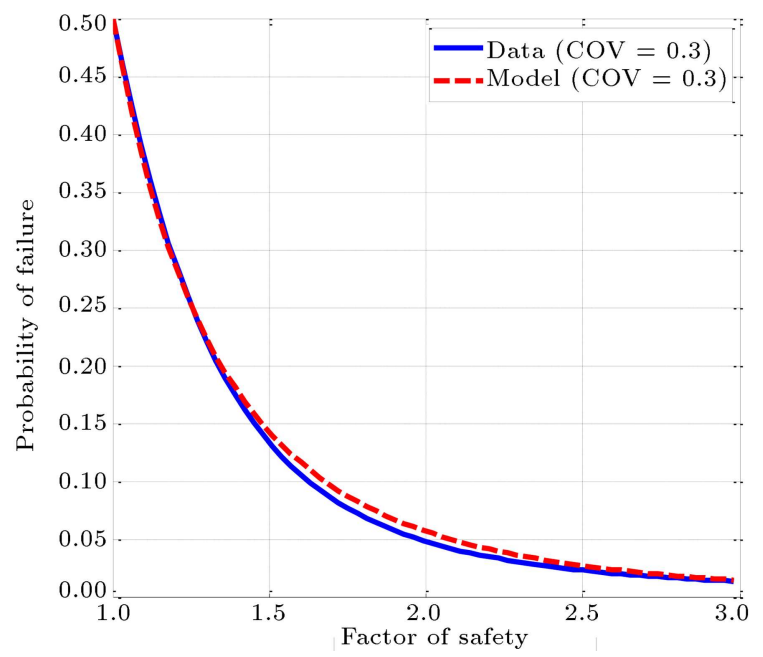

Figure 15. Probability of failure versus factor of safety at $\mathrm{COV}=0.3$.

concept of probability of failure, as discussed in section $6.1[34,35]$.

To demonstrate the accuracy of the proposed model in determining probability of failure, the GEP model prediction and actual data at $\mathrm{COV}=0.3$ are shown in Figure 15. Comparison of the curves indicates good performance of the proposed model for predicting the slope probability of failure. Furthermore, Table 6 presents the performance of the developed GEP model's prediction in detail.

\section{Conclusion}

Deterministic and probabilistic unsaturated slope stability analyses were investigated using Bishop's method and GEP model of SWCC. The suction of soil in various zones of slope was estimated using GEP model based on their water content. In this research, input parameters of SWCC, the effective internal angle of friction, effective cohesion, and unit weight of soil all were selected as uncertain soil parameters in analyses. In several stochastic analyses, the influence of the SWCC and its uncertainty was studied in slope stability analysis. Finally, the probability of failure was studied as a 3D surface whose dimensions are probability of failure, factor of safety, and COV of safety factor. A simple function was suggested using GEP to model this surface and predict the probability of failure in unsaturated slopes. Using the proposed model, the probability of failure of slopes can be assessed directly using deterministic factor of safety and its COV.

However, this paper has some simplified assumptions to carry out unsaturated slope stability such as neglecting pore air pressure and assuming specified water content profile in the slope. It would be desirable to determine water content profile in the slope using permeability function considering rainfall flux and evaporation. In such cases, the uncertainty of permeability function could be the field of further studies.

\section{References}

1. Tarantino, A. and El Mountassir, G. "Making unsaturated soil mechanics accessible for engineers: Preliminary hydraulic-mechanical characterisation stability assessment", Eng. Geol., 165, pp. 89-104 (2013).

2. Fredlund, D.G. and Rahardjo, H., Soil Mechanics for Unsaturated Soils, John Wiley \& Sons (1993).

3. Bergardo, D.T. and Anderson, L.R. "Stochastic analysis of pore pressure uncertainty for the probabilistic assessment of the safety of earth slopes", Soils Found, 25(2), pp. 85-105 (1985).

4. Gui, S., Zhang, R., Turner, J.P., and Xue, X. "Probabilistic slope stability analysis with stochastic soil hydraulic conductivity", J. Geotech. Geoenvironmental Eng., 126(1), pp. 1-9 (2000).

5. Sivakumar Babu, G.L. and Murthy, D.S. "Reliability analysis of unsaturated soil slopes", J. Geotech. Geoenvironmental Eng., 131(11), pp. 1423-1428 (2005).

6. Wolff, T.F. "Probabilistic slope stability in theory and practice", Uncertainty in the Geologic Environment from Theory to Practice, pp. 419-433 (1996).

7. U.S. Army Corps of Engineers, Risk-Based Analysis in Geotechnical Engineering for Support of Planning Studies, Department of the Army, Washington, DC (1998).

8. Ali, A., Huang, J., Lyamin, A.V., Sloan, S.W., Griffiths, D.V., Cassidy, M.J., and Li, J.H. "Simplified quantitative risk assessment of rainfall-induced landslides modelled by infinite slopes", Eng. Geol., 179, pp. 102-116 (2014).

9. Cho, S.E. "Probabilistic stability analysis of rainfallinduced landslides considering spatial variability of permeability", Eng. Geol., 171, pp. 11-20 (2014).

10. Zhang, J., Huang, H.W., Zhang, L.M., Zhu, H.H., and Shi, B. "Probabilistic prediction of rainfall-induced slope failure using a mechanics-based model", 168, pp. 129-140 (2014). 
11. Ng, C.W. and Menzies, B., Advanced Unsaturated Soil Mechanics and Engineering, CRC Press (2007).

12. Bishop, A.W. and Morgenstern, N. "Stability coefficients for earth slopes", Geotechnique, 10(4), pp. 129153 (1960).

13. Johari, A. and Hooshmandnejad, A. "Prediction of soil-water characteristic curve using gene expression programming", Iran. J. Sci. Technol. Trans. Civ. Eng., 39(C1), pp. 143-165 (2015).

14. "SoilVision 2002, SoilVision System Ltd., Sask., Saskatchewan."

15. Green, W.H. and Ampt, G. "Studies of soil physics, Part I - The flow of air and water through soils", Agric Sci, 4, pp. 1-24 (1911).

16. Richards, L.A. "Capillary conduction of liquids through porous mediums", Phys., 1, pp. 318-333 (1931).

17. Simunek, J., Van Genuchten, M., and Sejna, M. "The Hydrus-1D software package for simulating the movement of water, heat, and multiple solutes in variably saturated media, Version 4.16, HYDRUS Software Series 3", Dep. Environ. Sci. Univ. Calif. Riverside Riverside Calif. USA, p. 340 (2013).

18. Kennedy, J. "Particle swarm optimization", in Encyclopedia of Machine Learning, C. Sammut and G.I. Webb, Eds. Springer US, pp. 760-766 (2010).

19. Poli, R., Kennedy, J., and Blackwell, T. "Particle swarm optimization", Swarm Intell., 1(1), pp. 33-57 (2007).

20. Kennedy, J. and Eberhart, R. "Particle swarm optimization", in IEEE International Conference on Neural Networks, Proceedings, 4, pp. 1942-1948 (1995).

21. Griffiths, D.V. and Lu, N. "Unsaturated slope stability analysis with steady infiltration or evaporation using elasto-plastic finite elements", Int. J. Numer. Anal. Methods Geomech., 29(3), pp. 249-267 (2005).

22. Griffiths, D.V., Huang, J., and Fenton, G.A. "Influence of spatial variability on slope reliability using 2-D random fields", J. Geotech. Geoenvironmental Eng., 135(10) (2009).

23. Faradonbeh, R.S., Armaghani, D.J., Monjezi, M., and Mohamad, E.T. "Genetic programming and gene expression programming for flyrock assessment due to mine blasting", Int. J. Rock Mech. Min. Sci., 88, pp. 254-264 (2016).

24. Alkroosh, I. and Nikraz, H. "Predicting pile dynamic capacity via application of an evolutionary algorithm", Soils Found., 54(2), pp. 233-242 (2014).

25. Mollahasani, A., Alavi, A.H., and Gandomi, A.H. "Empirical modeling of plate load test moduli of soil via gene expression programming", Comput. Geotech., 38(2), pp. 281-286 (2011).

26. Johari, A., Javadi, A.A., and Najafi, H. "A genetic_based model to predict of maximum lateral displacement of retaining wall in granular soil", Sci. Iran., 23(1), pp. 54-65 (2016).
27. Taormina, R. and Chau, K.-W. "Data-driven input variable selection for rainfall-runoff modeling using binary-coded particle swarm optimization and extreme learning machines", J. Hydrol., 529(3), pp. 1617-1632 (2015).

28. Wu, C.L., Chau, K.W., and Li, Y.S. "Methods to improve neural network performance in daily flows prediction", J. Hydrol., 372(1-4), pp. 80-93 (2009).

29. Chen, X.Y., Chau, K.W., and Busari, A.O. "A comparative study of population-based optimization algorithms for downstream river flow forecasting by a hybrid neural network model", Eng. Appl. Artif. Intell., 46(A), pp. 258-268 (2015).

30. Ferreira, C. "Gene expression programming: a new adaptive algorithm for solving problems", ArXiv Prepr. Cs0102027 (2001).

31. Javankhoshdel, S. and Bathurst, R.J. "Simplified probabilistic slope stability design charts for cohesive and c- $\varphi$ soils", Can Geotech J, 51(9), pp. 1033-1045 (2014).

32. Phoon, K.-K. and Kulhawy, F.H. "Characterization of geotechnical variability.", Can Geotech J, 36(4), pp. 612-624 (1999).

33. GEPSOFT. GeneXproTools. Version 4.0. Available online: http://www.gepsoft.com (2006).

34. Johari, A. and Khodaparast, A.R. "Modelling of probability liquefaction based on standard penetration tests using the jointly distributed random variables method", Engineering Geology, 158, pp. 1-14 (2013).

35. Johari, A., Mousavi, S., and Hooshmand Nejad, A. "A seismic slope stability probabilistic model based on Bishop's method using analytical approach", Scientia Iranica, 22(3), pp. 728-741 (2015).

\section{Appendix}

Here, an example is presented to illustrate the procedure for prediction of SWCCs, using GEP model. For this purpose, the laboratory results of a sample were employed with the following input values: Void ratio $=$ 0.71 ; Initial water content $=26.89 \%$; Clay content $=$ $28.71 \%$; Silt content $=63.57 \%$. The value of suctions was started from $0.2 \mathrm{kPa}$ and multiplied by 2 until $1638.4 \mathrm{kPa}$. The SWCC is shown in Figure 2 and the calculation procedure is as follows:

1. The input parameters of the model were normalized to lie in an interval of $[0,1]$, using a max-min approach. The results are shown in Table A.1.

$$
\begin{aligned}
& e=\frac{e_{1}-e_{\min }}{e_{\max }-e_{\min }}=\frac{0.710-0.458}{2.846-0.458}=0.107 \\
& \omega_{0}=\frac{\omega_{0}-\omega_{0 \min }}{\omega_{0 \max }-\omega_{0 \min }}=\frac{26.89-17.34}{105.41-17.34}=0.108
\end{aligned}
$$


Table A.1. GEP model's predictions for selected sample.

\begin{tabular}{ccccccccc}
\hline $\boldsymbol{e}$ & $\boldsymbol{\omega}_{\mathbf{0}}$ & $\mathbf{C l}$ & $\mathbf{S i}$ & $\mathbf{S u}$ & $\begin{array}{c}\text { Experiment } \\
\text { water } \\
\text { content }\end{array}$ & $\begin{array}{c}\text { Predicted water } \\
\text { content } \\
\text { (normalized) }\end{array}$ & $\begin{array}{c}\text { Predicted water } \\
\text { content } \\
\text { (denormalized) }\end{array}$ & $\overline{\boldsymbol{\omega}}$ \\
\hline 0.107 & 0.108 & 0.335 & 0.689 & 0 & 0.248 & 0.255 & 0.252 & 0.248 \\
0.107 & 0.108 & 0.335 & 0.689 & 0.052 & 0.246 & 0.250 & 0.247 & 0.243 \\
0.107 & 0.108 & 0.335 & 0.689 & 0.105 & 0.244 & 0.246 & 0.243 & 0.239 \\
0.107 & 0.108 & 0.335 & 0.689 & 0.157 & 0.238 & 0.241 & 0.238 & 0.234 \\
0.107 & 0.108 & 0.335 & 0.689 & 0.210 & 0.231 & 0.236 & 0.233 & 0.229 \\
0.107 & 0.108 & 0.335 & 0.689 & 0.263 & 0.225 & 0.230 & 0.227 & 0.224 \\
0.107 & 0.108 & 0.335 & 0.689 & 0.315 & 0.217 & 0.223 & 0.221 & 0.217 \\
0.107 & 0.108 & 0.335 & 0.689 & 0.368 & 0.205 & 0.214 & 0.212 & 0.209 \\
0.107 & 0.1084 & 0.335 & 0.689 & 0.421 & 0.196 & 0.204 & 0.202 & 0.199 \\
0.107 & 0.1084 & 0.335 & 0.689 & 0.473 & 0.181 & 0.192 & 0.190 & 0.187 \\
0.107 & 0.1084 & 0.335 & 0.689 & 0.526 & 0.172 & 0.177 & 0.176 & 0.173 \\
0.107 & 0.1084 & 0.335 & 0.689 & 0.578 & 0.148 & 0.161 & 0.142 & 0.157 \\
0.107 & 0.1084 & 0.335 & 0.689 & 0.631 & 0.130 & 0.143 & 0.125 & 0.123 \\
0.1071 & 0.1084 & 0.335 & 0.689 & 0.684 & 0.120 & 0.125 & & \\
\hline
\end{tabular}

$$
\begin{aligned}
& \mathrm{Cl}=\frac{\mathrm{Cl}_{1}-\mathrm{Cl}_{\min }}{\mathrm{Cl}_{\max }-\mathrm{Cl}_{\min }}=\frac{28.71-4.4}{76.7-4.4}=0.336, \\
& S i=\frac{\mathrm{Si}_{1}-\mathrm{Si}_{\min }}{\mathrm{Si}_{\max }-\mathrm{Si}_{\min }}=\frac{63.57-10.3}{87.5-10.3}=0.689 .
\end{aligned}
$$

The magnitudes of $e, \omega_{0}, \mathrm{Cl}$ and $\mathrm{Si}$ were constant in the sample, while the magnitude of $\mathrm{Su}$ varied. For each arbitrary suction point, for instance $25.6 \mathrm{kPa}$, the normalization was done as follows:

$$
\begin{aligned}
\mathrm{Su} & =\frac{\log \left(S u_{1} / 100\right)-\log \left(S u_{\min } / 100\right)}{\log \left(S u_{\max } / 100\right)-\log \left(S u_{\min } / 100\right)} \\
& =\frac{\log (25.6 / 100)-\log (0.2 / 100)}{\log (104857.6 / 100)-\log (0.2 / 100)}=0.368
\end{aligned}
$$

2. By placing the above normalized value into Eq. (6), the result will be:

$$
\begin{aligned}
\omega_{(\text {Normilized })}= & 0.215 \\
\omega_{(\text {Denormalized })}= & 0.250 \times(0.9827-0.0018) \\
& +0.0018=0.213 .
\end{aligned}
$$

3. Based on the results of steps 2 and 3, the denormalized water content for suction of $25.6 \mathrm{kPa}$ is justified based on the initial experimental and predicted water content as follows:

$$
\bar{\omega}=0.252 \times \frac{0.248}{0.252}=0.209 .
$$

\section{Biographies}

Ali Johari obtained his $\mathrm{BS}, \mathrm{MS}$, and $\mathrm{PhD}$ degrees in 1995, 1999, and 2006, respectively, from Shiraz University, Iran, where he is currently an Assistant Professor in the Civil and Environmental Engineering Department. He was a Post-Doctoral Researcher at Exeter University in 2008, where he is also a member of the research staff of the Computational Geomechanics Group. His research interests include unsaturated soil mechanics, application of intelligent systems in geotechnical engineering, probabilistic models and reliability assessment. He has also consulted and supervised numerous geotechnical projects.

Ahmad Hooshmand Nejad was born in 1988 in Shiraz, Iran. He received his BS in Civil Engineering from Shahid Bahonar University of Kerman in 2011, Kerman, Iran and his MS degree from Shiraz University of Technology, Iran in 2015. His main research interests include unsaturated soil mechanics, slope stability, application of soft computing techniques in geotechnical engineering, probabilistic analysis and reliability assessment in geotechnical engineering. $\mathrm{He}$ has several publications in these fields.

Sobhan Mousavi was born in Behbahan, Iran in 1989. He obtained his BS degree in Civil Engineering from Yasuj University, Yasuj, Iran in 2012 and graduated from Shiraz University of Technology in MS degree in 2015. His current interests of research are about slope stability analysis, reliability and probabilistic analysis in geotechnical engineering. 